focus on German Studies.

Issue 28 (2021)

Book Review

Zeit und sâventiurer in Wolframs von Eschenbach >Parzivalı. Zur narrativen

Identitätskonstruktion des Helden

by Antje Sablotny, Berlin/Boston: de Gruyter, 2020 (Deutsche Literatur. Studien und

Quellen, vol. 34). 315 pp. \$114,99.

Michael Berger

Universität Wien

How to Cite: Stephan, Sigmund Jakob-Michael. "Zeit und saventiure in Wolframs von Eschenbach >Parzivak. Zur narrativen Identitätskonstruktion des Helden by Antje Sablotny”. focus on German Studies 28, no. 28, 2021, pp. 203-210. DOI:

10.34314/FOGS2021.00014. 


\section{Zeit und sâventiurer in Wolframs von Eschenbach >Parzival. Zur narrativen Identitätskonstruktion des Helden}

by Antje Sablotny, Berlin/Boston: de Gruyter, 2020 (Deutsche Literatur. Studien und Quellen, vol. 34). 315 pp. \$114,99.

Michael Berger

Wolframs von Eschenbach Parzival ist einer jener Texte, welche die Germanistik seit ihren Anfängen ungebrochen beschäftigt haben; dementsprechend unüberschaubar ist die Flut an Sekundärliteratur mittlerweile geworden. Dass Wolframs Werk mit seiner komplexen Struktur jedoch noch immer äußerst fruchtbar für die mediävistische Erzählforschung sein kann, beweist Antje Sablotnys vorliegende Monographie, die 2017 an der Technischen Universität Dresden als Dissertation angenommen wurde. Wie der Titel bereits nahelegt, geht es der Autorin um die Untersuchung der zeitlichen Organisation als auch um das Prinzip der âventiure als narrative Konstituenten für Parzivals Identität.

Einleitend verortet Sablotny ihre Arbeit, die ausdrücklich kein eigenes Instrumentarium ausbilden, sondern die Erzählordnung des Parzival anhand etablierter narratologischer Modelle erarbeiten möchte (19), im theoretischen Diskurs. Die Autorin beruft sich dabei hauptsächlich auf Gérard Genettes Erzähltheorie, deren Potential für die Analyse des Parzival sie in ihrer discours-Zentriertheit sieht (19f.). Als ebenso wichtig erweist 
sich Paul Ricœurs Konzept der narrativen Identität: Diese ergebe sich aus dem aporetischen Verhältnis von phänomenologischer und universeller Zeit, das erst durch die Etablierung einer Erzählordnung verhandelt werden könne (21f.). Das dritte für diese Arbeit zentrale theoretische Konzept ist Niklas Luhmanns religiöse Kommunikation, die sich durch das kontinuierliche Abarbeiten an der Kluft zwischen Immanenz und Transzendenz auszeichne (26). Dabei übernimmt Sablotny den im Rahmen des SFB 804 „Transzendenz und Gemeinsinn“ erarbeiteten, sehr weit gefassten Transzendenzbegriff, der sich nicht zwingend auf etwas Außerweltliches, sondern viel allgemeiner auf die „Konstruktion von Unverfügbarkeit“ beziehe (25f.). Damit schafft es die Autorin auf geschickte Weise, die Betrachtung der Transzendenz auch losgelöst von der heilsgeschichtlichen Komponente, die in der Paræival-Forschung wiederholt ins Zentrum gerückt wurde, zu untersuchen.

Im zweiten Teil liegt der Fokus auf der programmatischen Entfaltung der komplexen Zeitstrukturierung durch den Erzähler. So drücke der Prolog vor allem die Nicht-Linearität des diskursiven Erzählaktes aus, und zwar nicht nur durch die zeitlich enthobene Sprecherposition, von der aus zugleich nach vorne (auf das Eræä̈hlen der Geschichte) als auch nach hinten (auf das Sich-Ereignen des Geschehens) geblickt werde (36f.). Dass diese zeitliche Verortung als „poetologische[s] Prinzip“ (38) des Parįival gesehen werden könne, wird zudem an den rätselhaften Metaphern Wolframs - das vliegende bîspel, der schellec hase, zin anderhalp ame glase oder des blinden truom - expliziert: Sie alle stehen 
laut Sablotny für die Nicht-Linearität des Wolfram'schen Erzählens ein, indem sie narrative Strategien der Zeitorganisation vorwegnehmen (44f.). Im Anschluss daran liest sie das Bogengleichnis als Versinnbildlichung der zeitlichen Organisation der âventiure, die der Erzähler zur - wortwörtlichen - „Spannungserzeugung“ (48) steuern könne; Kyôt, auf dessen Wunsch Wolfram an dieser Stelle noch substantielle Informationen zurückhalten müsse, personifiziere hierbei gleichsam das erzählerische Prinzip selbst (51). Die Argumentation schafft die letztlich wohl unmögliche Herkulesaufgabe, das poetologische Rätsel des Bogengleichnisses restlos aufzulösen, nicht ganz, bietet aber dennoch einen sehr überzeugenden textimmanenten Ausgangspunkt für die nachfolgende Analyse der Erzählstruktur.

Im dritten Kapitel wendet sich Sablotny der Untersuchung der Aspekte ,Zeit' und ,âventiure‘ zu. Zunächst wird die narrative Produktion von zeitlicher Dauer beschrieben, die für die Einordnung der erzählten Zeit im Parzival weit wichtiger sei als die Rekonstruktion präziser chronologische Abfolgen (62f.). Dies erreiche Wolfram u. a. durch die Inszenierung von Gleichzeitigkeit, die nicht nur im Gâwân-Teil, sondern in allen direkt erzählten oder nur referierten Nebenhandlungen zum Einsatz komme; erst durch die Synchronisation dieser Einzelepisoden komme die ,Geschichte‘ Parzivals, die Identität des Helden, zustande (66).

Der âventiure-Begriff hingegen erweist sich für Sablotny aufgrund seiner „semantische[n] Offenheit“ (110) als praktikables Scharnier zwischen bistoire und discours: Der „Kreislauf 
von âventiure-Handeln und âventiure-Erzählen“ (Peter Strohschneider) sei als „,autopoietisches Element des sozialen Systems Artushof“ zu greifen, wodurch die âventiure zum Kommunikationsinstrument für das soziale Gefüge des Artushofes als auch für das System des höfischen Erzählens avanciere (94). Im Parzival allerdings sei ein semantischer Wandel des arthurischen âventiure-Konzeptes zu erkennen, der sich etwa durch die Infragestellung der „Dienst-Lohn-Logik“ (119), durch die Unmöglichkeit der aktiven Fehlerkompensation (125) oder durch die Ironisierung des Zweikampfes in der Blutstropfenszene (131ff.) bemerkbar mache. Nicht zuletzt der Auftritt der personifizierten vrou Âventiure bezeuge schließlich den Status der âventiure als „Schnittstelle zwischen intradiegetischer und extradiegetischer Ebene“ (148), denn erst durch die organisierte Erzählung der âventiure werde diese letztlich konstituiert (135).

Der vierte Teil der Arbeit widmet sich dem Erzählen von Transzendenz, wofür Sablotny die augustinische Zeitvorstellung, welche die Gotteserkenntnis im Spannungsfeld von Zeitlichkeit und Ewigkeit positioniert (162f.), und Ricœurs Idee der zeitlichen Einheitsstiftung im Zuge der Narration durch die Hilfsmittel ,Kalender‘, ,Generationenfolge` und ,Spur‘, die eine Verknüpfung von phänomenologisch-subjektiver und universell-objektiver Zeit zu leisten vermögen (175ff.), aufruft. Auf dieser Basis präsentiert sie den Kyôt-Exkurs sehr schlüssig als Nachzeichnung einer „Genese der Dichtung aus der Transzendenz“ (190): Das in der Schilderung des transzendenten 
Ursprunges und der mehrmaligen narrativen Vermittlung der Gralsgeschichte hergestellte „Spannungsverhältnis von Sukzession und Simultaneität" (205) ermögliche eine Teilhabe der Erzählung an der Transzendenz; das metaleptische Potential der Grals-âventiure (195) werde somit „,vermittels der Dekonstruktion zeitlicher Strukturen erfahrbar gemacht“ (213).

Überzeugend gelingt auch die Herausarbeitung der Produktion von Präsenz (Hans Ulrich Gumbrecht). So vermöge es etwa die Gralsprozession, durch narrative Mittel wie Nullfokalisierung und Drosselung des Erzähltempos ein Spannungsfeld von Präsenz und Absenz des Göttlichen zu erzeugen, das eine Transzendenzerfahrung des Helden lediglich als abgewiesene Alternative in den Raum stelle (266ff.). In der komplementären Blutstropfenszene hingegen diene die Außenperspektive im Sinne eines „,negative[n] Erzählen[s]“ der Verdeutlichung der „Zeitenthobenheit und Transzendenzerfahrung des Helden“ (241). Parzivals Abwesenheit im Gâwân-Teil schließlich fasst Sablotny als Spiegelung der in der Blutstropfenszene evidenten narrativen Abwesenheit Parzivals ,auf der Makroebene des Romans“ (252): Während sie im Falle der ersten Abwesenheit durchaus mit der Forschung übereinstimmt und sie als Ausdruck von Parzivals Gottesferne sieht, liest sie die zweite Abwesenheit als „göttliche Transzendenzerfahrung“ (257).

Im letzten Kapitel betont die Autorin noch einmal, inwiefern die Identitätskonstruktion Parzivals nicht durch den Helden selbst, sondern durch Zuschreibungen von außen geschieht. Zu diesem Zweck konzentriert sie sich auf drei 
„Figuren der Zeitordnung“ (262) - Trevrizent, Sigûne und Cundrîe -, denen Parzival mehrmals begegnet und die seine Erlebnisse je im Nachhinein rekapitulieren und somit seine Geschichte mit der Geschichte des Grals in Eins führen (262f.). Indem Wolfram diese metadiegetischen Erzählerfiguren einführt, die retrospektiv eine zeitliche Ordnung herstellen und somit Parzivals Geschichte in der histoire konstruieren, manifestiere sich noch einmal „die konstitutive Reziprozität von Zeit und âventiure für den Parzivival und die Identität seines Helden“ (288). Wenn es der Autorin in den ersten Kapiteln auch nicht immer ganz gelingt, die Zielgerichtetheit des Arguments permanent aufrechtzuerhalten, so zeigt sich spätestens hier in aller Deutlichkeit, wie die scheinbar nur weitläufig miteinander verwandten Faktoren ,Zeit' und âventiure` bei genauerer Betrachtung doch untrennbar miteinander verknüpft sind.

Das erklärte Ziel von Sablotnys Arbeit ist es - und muss es aufgrund des enormen Umfanges der Forschungstradition auch sein -, auf bereits etablierten Ergebnissen (exemplarisch genannt seien Susanne Knaeble, Marina Münkler, Katharina Philipowski, Bernd Schirok und Hans-Hugo Steinhoff) aufzubauen und somit von einem bereits sehr profunden Ausgangspunkt aus noch tiefer in die Materie einzutauchen, was aufgrund der daraus resultierenden (auch sprachlichen) Komplexität zeitweise etwas sperrig wirkt. Auch die recht ausführlichen Forschungsreferate, welche die ganze Arbeit durchziehen, belegen zwar die Expertise der Verfasserin, stören aber bisweilen die Stringenz des meist sehr 
elaborierten Arguments. Doch in der Zusammenschau der Ergebnisse Sablotnys wiegen diese Kritikpunkte nicht allzu schwer, schafft sie es mit ihrer Studie doch, einen letztlich sehr erhellenden Beitrag zur komplexen Erzählstruktur des Parzival zu liefern: Vor allem die Strategien der Identitätskonstruktion durch narrative Organisation und das metaleptische Potential der âventiure werfen neue Schlaglichter auf das bereits so intensiv erforschte Werk und schaffen interessante methodische Anknüpfungspunkte für die Beschäftigung mit mittelalterlicher Literatur. 